\title{
Enterocolitis, antimicrobianos y síndrome hemolítico urémico en niños: Revisión de un concepto
}

Patricio Herrera, Carlos Valenzuela, Marcos Skarmeta, Miguel Méndez y Álvaro Bustos-González

\section{Enterocolitis, antimicrobials and hemolytic-uremic syndrome in children: Review of a concept}

Background: Treatment of children with Escherichia coli O157:H7 enterocolitis could be related with risk of Hemolytic-Uremic Syndrome (HUS). The proscription of antibiotics use until cultures ruled out this infection has been proposed (Wong et al.). This concept became generalized causing dilemma in clinical decisions and research. The objective of this study was to review the evidence related with this topic. Methods: A 1999-2011 literature review of studies related with E.coli $\mathrm{O} 157: \mathrm{H} 7$, antibiotics and HUS in children. Results: We found one randomized controlled trial, three cohort studies, one case-control and one meta-analysis with pediatric data on the topic. After methodological and metrical analysis, five studies showed opposite results as compared toWong et al. All had statistical power enough to detect Wong's association but did not happen. Wong's study had no statistical power to detect risk ratios $\leq 11$. Conclusion: These results are neither reproducible nor generalizable, consequently clinical decisions should be solved following local epidemiology.

Key words: enterocolitis, antibiotics, hemolytic-uremic syndrome, complications, children.

Palabras clave: Enterocolitis, antimicrobianos, síndrome hemolítico urémico, niños.

\section{$\mathrm{L}$} as enterocolitis bacterianas (EBs) causadas por Escherichia coli, Shigella, Salmonella spp y otras, son un problema común en todo el mundo, cuyo significado difiere según factores como el área geográfica, grupo socioeconómico, y grupo etario afectado. En los países en desarrollo adquieren rasgos particulares por su alto riesgo y la insuficiencia de recursos para la identificación precoz del agente causal. Aunque el uso de antimicrobianos en EBs puede ser discutible en ciertas circunstancias, en determinados lugares puede ser indispensable ${ }^{1,2}$.

Datos publicados hace diez años por Wong C.S. y cols. ${ }^{3}$, que mostraron una fuerte asociación entre infecciones por E. coli $\mathrm{O} 157: \mathrm{H} 7$ tratadas con antimicrobianos y el desarrollo de síndrome hemolítico urémico (SHU), movió a sus autores a pronunciarse contra dar antimicrobianos a niños que podrían estar infectados con E. coli O157:H7 hasta que los resultados del coprocultivo informaran que el patógeno responsable es tratable apropiadamente con un antimicrobiano $^{3}$. Este estudio, presentado como un diseño analítico (estudio prospectivo de cohorte con cálculo de razones de riesgo), comunicó un riesgo relativo de 17,3 (intervalo de confianza del 95\% 2,2 a 137), magnitud que resultó amenazante para los pediatras, en especial de América Latina, y en particular para aquéllos que deben tomar decisiones en poblaciones donde las EBs son un problema prevalente y de primera magnitud. Particularmente en éstos-los recursos de laboratorio para la identificación lo suficientemente precoz de bacterias que no sean las más comunes no están a su alcance- el concepto de Wong CS y cols., suscitó un comentario inmediato ante la restricción propuesta, expresando la preocupación en cuanto a que el mensaje de este estudio se extendería prematuramente al tratamiento de las diarreas con sangre en todo el mundo y en lugares donde la prevalencia de Shigella spp y $E$. coli es similar y las cepas de otras E. coli no O157:H7 son comunes con lo que, contener el tratamiento antimicrobiano hasta saber la causa de la diarrea, podría tener más riesgos que beneficios ${ }^{4}$.

La preocupación de los autores de tal comentario parece haberse materializado y, pese a que han transcurrido más de diez años desde la afirmación de Wong C.S. y cols. ${ }^{3}$, la noción que involucra al tratamiento de las diarreas en niños con antimicrobianos como responsable de un alto riesgo de SHU, se ha convertido en un lugar común ${ }^{5-11}$, lo que dificulta la toma de decisiones pediátricas e impide formular proyectos de ensayos clínicos controlados (ECCs) que pudieran aportar información sólida sobre este y otros aspectos relacionados con las enterocolitis, SHU y el uso de antimicrobianos en niños. Una revisión previa (no publicada) ${ }^{12}$ no encontró evidencias que sustentaran la proscripción de antimicrobianos en diarreas (con o sin sangre) causada por E. coli O157:H7.

\section{Objetivos}

Examinar si existe nueva información que permita ampliar el conocimiento del problema y justificar o no
Universidad de Chile, Santiago. Facultad de Medicina, Campus Norte. Departamento de Pediatría ( $\mathrm{PH}$, MS). Instituto de Ciencias Biomédicas (CV).

Servicio de Salud Metropolitano Norte. Santiago, Chile. Hospital Roberto Del Río (MM). Universidad Javeriana Bogotá, Colombia.

Facultad de Ciencias de la Salud de la Universidad del Sinú, Montería, Colombia (AB).

Los autores declaran no tener conflictos de interés

Recibido: 11 de septiembre de 2011 Aceptado: 3 de abril de 2012

Correspondencia a: Patricio Herrera Labarca pherrera@vtr.net. 
la generalización a que dio lugar el artículo de Wong y cols. ${ }^{3}$, tanto para resolver el dilema en la práctica clínica pediátrica, que implica enfrentar las enterocolitis y las otras etiologías de $\mathrm{SHU}^{5}$, así como conocer el estado del problema ético que planteó desde entonces ("equipoise" desbalanceado) ${ }^{13}$ al establecer un cuestionamiento a la investigación sobre el tema, en particular el uso de diseños intervencionistas. Nuestros objetivos no buscan preconizar el uso sistemático de antimicrobianos en diarreas de niños.

\section{Métodos}

Siguiendo los métodos estándares ${ }^{14}$ para revisiones clínicas, realizamos una búsqueda de estudios identificados por los términos "Escherichia coli O157:H7", "antibiotics", "hemolytic-uremic syndrome" y "children all", 0 a 18), por medio de PubMed, ScIELO y la base de datos de Cochrane Library para ECCs, durante el período arbitrario de 30 de junio de 1999 a 31 de marzo de 2011, además de proceder a la selección manual de artículos a partir de las listas bibliográficas de los trabajos seleccionados en que se informara de los datos necesarios para el estudio en niños. Usamos el término "enterocolitis", omitiendo la condición "con sangre" para salvar la variedad de términos empleados en la literatura y porque este último no es un rasgo siempre presente en la población de interés. Tres de nosotros (PH, CV, MS) clasificamos los artículos en alguna de las siguientes categorías: ECC, cohortes prospectivas, caso-control, estudio de una cohorte y series de casos, siguiendo métodos de análisis metodológico estándares ${ }^{15,16}$, complementado con un análisis crítico de los diseños utilizados, además de examinar sus correspondientes métodos de análisis estadístico. Las diferencias entre los participantes se resolvieron por consenso.

El análisis métrico de los resultados se limitó a usar los resultados de interés primario (la asociación mencionada) usando los datos "crudos" proporcionados en los estudios seleccionados, como ha sido propuesto por otros autores $^{17}$, ordenados en tablas de 2 × 2 , excluyendo otras manipulaciones bioestadísticas. Se calcularon las medidas de asociación como razón de disparidad (OR) cuando los resultados del estudio exhibieron como "casos" los niños con SHU (análisis de la probabilidad de exposición: antimicrobianos) y riesgo relativo (RR) cuando los "casos" fueron los niños que recibieron antimicrobianos (análisis de la probabilidad de efecto: SHU), ambos con sus respectivos intervalos de confianza del 95\% (IC 95\%). Para analizar el primer objetivo, se calculó el poder estadístico de los estudios para detectar la medida de asociación (OR o RR) correspondiente al tipo de diseño declarado por los autores o discernible de la forma de ordenar y presentar los resultados. En ausencia de hipótesis en el texto, para calcular el poder de cada estudio a partir del tamaño de la muestra y la razón casos:controles resultante de las tablas, se estableció el nivel de error alfa en 0,05 bilateral previendo que, en ausencia de hipótesis formal a priori, la dirección de la asociación pudiera ser en cualquiera de las dos direcciones, independientemente de la resultante en cada estudio.

Dada la magnitud inusitadamente elevada de la asociación del estudio de Wong C.S. y cols. ${ }^{3}$, centramos los análisis para responder las preguntas.

- si este estudio tenía poder estadístico para detectar asociaciones de menor magnitud que la encontrada y

- si los otros estudios tenían tal poder para detectar asociaciones del tamaño del estudio de Wong C.S. y cols. ${ }^{3}$.

Usamos el programa de Dupont WD and Plummer WD "PS Power and Sample Size" Ver. 3.0.14 con este fin, usando los valores de Po, n, m, y razones de riesgo extraídos de los datos en el texto, según se ha descrito más arriba, y de las tablas de los artículos seleccionados.

\section{Resultados}

Encontramos nueve publicaciones relacionadas con el tema que nos ocupa ${ }^{18-25}$, además de un meta-análisis sobre el tema ${ }^{26}$. Cuatro de éstos fueron descartados por no contener datos de niños o éstos eran insuficientes para el análisis. El meta-análisis fue estudiado aparte.

El material estudiado consistió en un $\mathrm{ECC}^{20}$, tres estudios de una cohorte ${ }^{13,18,19}$ y un caso-control ${ }^{22}$, además del meta-análisis sobre el tema ${ }^{26}$. El estudio de Wong y cols. ${ }^{3}$, fue analizado como una cohorte dividida en dos grupos, a modo de comparación de cohortes incipientes, y como caso-control, según se presentaron las categorías de SHU (Tabla 1 de Wong y cols.) o las categoría de exposición a antimicrobianos (Tabla 2 de Wong y cols.), respectivamente. Las características de los estudios analizados aparecen en la Tabla 1.

Es de hacer notar que, salvo el ECC, se observó la transformación -por parte de los autores de los estudiosde series de casos (que pueden entenderse como una sola cohorte) por dicotomía de la serie en dos categorías o grupos, a menudo según varios criterios para, de allí y sin hipótesis, proceder al cálculo de razones de riesgo, pero no comparando dos cohortes incipientes y sus controles apropiados (en todo comparables, excepto en la variable “exposición" o la "efecto", según la dirección del diseño) siguiendo una hipótesis precisa previa y explícita. Esto aplica a los estudios presentados como "cohortes" y como "caso-control".

La falta de hipótesis y el desarrollo de diseños metodológicamente más sólidos pueden explicar, en parte, la falta de significación estadística de los estudios de Bell y cols. ${ }^{17}$, y Proulx y cols. ${ }^{20}$. A diferencia del resultado de Wong y cols., todos los estudios sugirieron efecto "protector" de los antimicrobianos ${ }^{19,21,22}$ o ningún efecto ${ }^{18}$ sobre la probabilidad de SHU, como lo muestra el estudio de Safdar y cols. ${ }^{26}$. Más importante, todos los estudios 
con razones de riesgo $\leq 1$ tuvieron poder estadístico para detector RR y ORs superiores a 3 y, por ello, magnitud de razones de riesgo que incluyen las de Wong y cols. ${ }^{3}$ (Tabla 2). El meta-análisis ${ }^{26}$ no logró combinar resultados por la heterogeneidad de los grupos de estudio pero el resultado de su análisis, aunque concuerda con nuestra apreciación en el sentido que no existe sustento para la afirmación que proscribe el uso de antimicrobianos por alto riesgo de SHU, entrega un "OR sumario", "u OR resumen" (del inglés "pooled OR") que no tiene significación si las pruebas de heterogeneidad demuestran que los "OR sumados" son estadísticamente diferentes $(\mathrm{p} \leq 0,05)^{27,28}$. Señala además la ausencia de sesgo de publicación, lo cual destaca la escasez de estudios sobre la asociación que nos preocupa, lo que es, hasta ahora, comprensible.

\section{Discusión}

Nuestra primera conclusión es que los resultados de Wong y cols., deben ser considerados como no generalizables ni reproducibles, ya que no es posible aceptar que todos los otros estudios - que, en conjunto, constituyen un número elevado de casos- entregaron resultados opuestos a los de Wong y cols., referidos a la asociación entre el riesgo de SHU y tratamiento con antimicrobianos de niños con infección por E. coli O157:H7. Más aún, se muestra cómo el estudio de Wong C.S. y cols., careció de poder estadístico para detectar razones de riesgo menores que las publicadas.

Una segunda conclusión es que, ya que complicaciones graves de las infecciones por $E$. coli no se restringen al SHU ni es ésta su única etiología, la normalización del "equipoise" puede permitir estudios intervencionistas a raíz de brotes de E. coli $\mathrm{O} 157: \mathrm{H} 7$, porque en éstos puede suponerse una monoclonalidad que facilita la identificación precoz de la etiología. Por otro lado, se necesitan diseños más robustos que series de casos de tamaño insuficiente para el estudio de tales brotes, más que la simple contemplación pasiva de este problema.

No intentamos un meta-análisis no sólo porque las pruebas de heterogeneidad, ya hechas por Safdar y cols., impidieron a éste lograrlo en el estricto sentido estadísti$\mathrm{co}^{26}$, sino porque la heterogeneidad va mucho más allá de consideraciones matemáticas o simplemente estadísticas y están determinadas, entre otros factores, como la ambigüedad de los diseños (con excepciones), la heterogeneidad de los grupos estudiados de acuerdo a la edad, los antimicrobianos usados, el origen de los casos (brotes vs grupos de casos esporádicos), diferencias de los objetivos, etc., factores que impiden combinar los resultados a no ser que el resultado puntual se lo controle en base de éstas y otras variables, como posibles confundidores ${ }^{27,28}$.

Una explicación de resultados contradictorios puede encontrarse en presentar series de casos eventualmente divididas en dos a posteriori -para calcular razones de
Tabla 1. Infección por Escherichia coli 0157:H7, antimicrobianos y SHU: datos de articulos seleccionados

\begin{tabular}{|c|c|c|c|c|c|}
\hline $\begin{array}{l}\text { Autor } \\
\text { (referencia\#) }\end{array}$ & Año & $\mathrm{n}$ & Tipo de estudio & $\begin{array}{c}\text { Razón de } \\
\text { Riesgo }\end{array}$ & IC $95 \%$ \\
\hline Proul $x^{21}$ & 1992 & 47 & Clinical controlled trial & $\mathrm{RR}=0,57$ & 0,09 a 3,46 \\
\hline Cimolai ${ }^{22}$ & 1990 & 101 & Caso-control "retrospectivo" & $\mathrm{OR}=0,35$ & 0,026 a 0,735 \\
\hline Bell ${ }^{18}$ & 1997 & 268 & "Cohorte retrospectiva" & $\mathrm{OR}=1,29$ & 0,33 a 1,38 \\
\hline |keda ${ }^{19}$ & 1999 & 130 & "Cohorte prospectiva" & $\mathrm{OR}=0,67$ & 0,03 a 0,78 \\
\hline Wong $^{3}$ & 2000 & 71 & "Cohorte prospectiva" & $\begin{aligned} O R & =14,3 \\
R R & =7,6\end{aligned}$ & $\begin{array}{l}2,14 \text { a } 93,8 \\
2,46 \text { a } 23,7\end{array}$ \\
\hline
\end{tabular}

(Razones de riesgo menores de1 implican efecto protector del antibiótico).

Tabla 2. Poder estadístico calculado de datos de los articulos seleccionados

\begin{tabular}{|c|c|c|c|c|c|c|}
\hline Autor & Po & $\underline{\mathbf{n}}$ & $\underline{\mathrm{m}}$ & OR & $\mathbf{R R}$ & Poder para detectar razón de riesgo \\
\hline Proul $x^{21}$ & 0,16 & 22 & 1,0 & & 0,57 & $>3^{*}$ \\
\hline Cimolai 22 & 0,58 & 28 & 2,6 & 0,35 & & $>5$ \\
\hline Bell18 & 0,13 & 50 & 4,4 & 1,29 & & $>3$ \\
\hline Ikeda $^{19}$ & 0,10 & 14 & 8,3 & 0,67 & & $>6$ \\
\hline \multirow[t]{2}{*}{ Wong et $\mathrm{al}^{3}$} & 0,07 & 10 & 6,1 & 14,3 & & $>11^{* *}$ \\
\hline & & & & & $(7,6)$ & $n / c$ \\
\hline
\end{tabular}

Diseños de estudio (referencias 10,11,12) tenían poder suficiente para detector $O R=14,3$. *Poder suficiente para detectar $\mathrm{RR}>3$. ${ }^{*}$ Sin poder para detectar $\mathrm{OR}<11$. $\mathrm{PO}=$ Valor bajo la hipótesis nula (Tablas $2 \times 2$ calculadas). $\underline{n}=$ Número de casos. $\underline{m}=$ Razón controles por caso. $\mathrm{n} / \mathrm{c}=$ No corresponde.

riesgo-y la ausencia de hipótesis operacionales, por lo que los resultados en tales instancias deben ser considerados como conclusiones débiles y no generalizables si se las compara con los resultados de estudios más robustos, como es el estudio comparando cohortes (que admite inferencia estadística) y el caso-control formal, ambos con hipótesis explícita y tamaños de muestra calculados para ser adecuados ${ }^{29}$. Por ello, la "modelación" estadística con base en los resultados de estudios como los analizados, parece no tener justificación o ser, al menos, controvertibles.

La necesidad de ECC ha sido propuesta por algunos autores $^{15,16}$, pero la proscripción basada en los resultados de Wong y cols., la han hecho inaceptable por el problema ético que surgió con el desequilibro del "equipoise", dando por sentado que el brazo de "tratados" estuviera -con certeza- expuesto a un grave daño.

Sin embargo, el uso de antimicrobianos en síndromes diarreicos en niños se ha convertido en un problema cada vez más complejo, ya que el espectro etiológico tiene estructuras diferentes de un lugar respecto a otros, así como el de las resistencias de los entero-patógenos a los antimicrobianos.

El que el tratamiento con antimicrobianos de las infecciones por Shigella esté justificado aun cuando el SHU puede ser una complicación por cepas toxigénicas, ilustra la necesidad de más estudios para reconsiderar el balance riesgo/beneficio de las terapias de diarreas en los diferentes niveles de atención. 
Mientras, las decisiones al respecto dependerán de la gravedad de los casos y el tipo de información local sobre las etiologías y resistencias bacterianas.

\section{Resumen}

Antecedentes: El tratamiento de niños con enterocolitis por Escherichia coli O157:H7 estaría relacionado con alto riesgo de síndrome hemolítico-urémico (SHU). Se propuso la proscripción del uso de antimicrobianos hasta que los cultivos descartasen esta etiología (Wong y cols.). Este concepto se generalizó, causando dilemas para las decisiones clínicas y la investigación. El objetivo de este estudio fue revisar la evidencia relacionada con este tema. Métodos: Se realizó una búsqueda en la literatura 1999-2011de estudios relacionados con Escherichia coli O157:H7, antimicrobianos, SHU y niños. Resultados: Encontramos un ensayo clínico controlado, tres estudios de cohortes, un caso-control con datos de niños y un metaanálisis. Al análisis metodológico y métrico, cinco estudios obtuvieron resultados opuestos al de Wong y cols. Todos tenían poder estadístico suficiente para detectar la medida de asociación de Wong y cols., pero ello no ocurrió. El estudio de Wong y cols. no tenía tal poder para detectar razones de riesgo $\leq 11$. Conclusión: Los resultados de Wong y cols.no son reproducibles ni generalizables, por lo que las decisiones clínicas debieran resolverse según la epidemiología local, sin restricciones.

\section{Referencias bibliográficas}

1.- Haltalin K C, Nelson J D, Ring R III, Sladoje M. Double-blind treatment study of shigellosis comparing ampicillin, sulfadiazine and placebo. J Pediatr 1967; 70: 970-81.

2.- Christopher P R H, David K V, John S M, Sankarapandian V. Antibiotic therapy for Shigella dysentery. Cochrane Database of Systematic Reviews 2010, Issue 8. Art. No.: CD006784. DOI: 10.1002/14651858. CD006784.pub4.

3.- Wong C S, Jelacic S, Habeeb R L, Watkins S L, Tarr PI. The risk of hemolytic-uremic syndrome after antibiotic treatment of Escherichia coli O157:H7 infections. New Engl J Med 2000; 342: 1930-36

4.- O'Ryan M, Prado V. Risk of hemolyticuremic syndrome after antibiotic treatment of Escherichia coli O157:H7 infections. New Engl J Med 2000; 343: 1271.

5.- Prado V, Cavagnaro F. Síndrome hemolítico urémico asociado a infección intestinal por Escherichia coli productora de shigatoxina (STEC) en pacientes chilenos: aspectos clínicos y epidemiológicos. Rev Chilena Infectol 2008; 25: 435-44.

6.- Farquhar D. E. coli, antibiotics and hemolyticuremic syndrome in children. Can Med Assoc J 2002; 163 (4): 438.

7.- Ibáñez J, Caletti MG. Antibióticos y severidad del fallo renal agudo en el síndrome hemolítico urémico". $34^{\circ}$ Congreso de la Sociedad Argentina de Pediatría, Oct. 2006, Córdova, Argentina.

8.- Hospital Roberto Del Río. Guías de Atención Pediátrica. 5a Ed. Mediterráneo, Santiago, 2005.

9.- Rivero M, Padola N L, Etcheverría A I, Parma A E. Escherichia coli enterohemorrágica y síndrome hemolítico urémico en Argentina. Medicina (BsAs, Argentina) 2004; 64: 352-6.

10.- Caja Seg. Soc. Direc. Med. Y Ter. "Tratamiento de diarreas agudas en niños y adultos en primer y segundo nivel" $2005 ; \mathrm{N}^{\circ} 35$.
11.- Iijima K, Kamioka I, Nozu K. Management of diarrhea-associated hemolytic uremic syndrome in children. Clin Exp Nephrol 2008; 12 (1): 16-9.

12.- Herrera P, Prado V. Shigatoxin-producing Escherichia coli infections, antibiotics and hemolytic uremic syndrome in children. Seeking for evidence. $3^{\text {er }}$ Congreso Mundial de Infectología Pediátrica. Santiago, Chile 19-23 de Noviembre, 2002. Book of Abstracts p. 85.

13.- Fries J F, Krishman E. Equipoise, design bias and CRT. The elusive ethics of new drug development. Arthritis Res Ther 2004; 6: R250-R255.

14.- Mulrow CD. The medical review article: State of the science. Ann Intern Med 1987; 106: 485-6.

15.- Sackett D L, Haynes R B, Guyatt G H, Tugwell P. Clinical Epidemiology: A Basic Science for Clinical Practice. Little Brown \& Co. Boston, 2nd. Ed. 1991.

16.- Sackett D, Straus Sharon E, Richardson W S, Rosemberg W, Haynes R B. Evidence-Based Medicine. How to practice and teach. Churchill Livingstone, UK, $2^{\text {nd }} E d .2000$.

17.- Aragon T, Fernyak S F, Reiter R. Risk of hemolytic-uremic syndrome after antibiotic treatment of Escherichia coli $\mathrm{O} 157: \mathrm{H} 7$ infections. New Engl J Med 2000; 343: 1271-2.

18.- Bell B P, Griffin P M, Lozano P, Christie D L, Kobayashi J M, Tarr P I. Predictors of hemolytic uremic syndrome in children during a large outbreak of Escherichia coli O157:H7 infections. Pediatrics 1997; 100/1/e12.

19.- Ikeda K, Ida O, Kimoto K, Takatorige T, Nakanishi N, Tatara K. Effect of early fosfomycin treatment on prevention of hemolytic uremic syndrome accompanying Escherichia coli $\mathrm{O} 157: \mathrm{H} 7$ infection. Clin Nephrol 1999; 52: 357-62.

20.- Slutzker L, Ries A A, Maloney K, Wells J G, Greene K D, Griffin P M. A Nationwide case- control study of Escherichia coli O157:H7 infections in the United States. J Infect Dis 1998; 177: 962-6.

21.- Proulx F, Turgeon J P, Delage G, Lafleur L, Chicoine L. Randomized controlled trial of antibiotic therapy for Escherichia coli O157:H7 enteritis. J Pediatr 1992; 121: 299-303.

22.- Cimolai N, Carter J E, Morrison B J, Anderson J D. Risk factors for the progression of Escherichia coli O157:H7 enteritis to hemolytic-uremic syndrome. J Pediatr 1990; 116: 589-92.

23.- Ostroff S M, Kabayashi J M, Lewis J H. Infections with Escherichia coli O157:H7 in Washington State: The first year of statewide surveillance. JAMA 1989; 262: 355-9.

24.- Dundas S, Todd W T A, Stewart A I, Murdoch P S, Chaudhuri A K, Hutchinson S J. The central Scotland Escherichia coli O157:H7 outbreak: Risk factors for the haemolytic-uremic syndrome and death among hospitalized patients. Clin Infect Dis 2001; 33: 923-31.

25.- Pavia A, Nichols C R, Green D P, Tauxe R V, Mottice S, Greene K D, et al. Hemolytic-uremic syndrome during an outbreak of Escherichia coli $\mathrm{O} 157: \mathrm{H} 7$ infections in institutions for mentally retarded persons: Clinical and epidemiological observations. J Pediatr 1990; 116: 544-51.

26.- Safdar N, Said A, Gangnon RE, Maki DG. Risk of hemolytic-uremic syndrome after antibiotic treatment of Escherichia coli $\mathrm{O} 157: \mathrm{H} 7$ infections: A meta analysis. JAMA 2002, 288 : 996-1001.

27.- Schlesslman J J. Case-control Studies. Oxford University Press N.Y. 1982.

28.- Kleinbaum D G, Kupper L L, Morgenstern H. Epidemiological research" Van Nostrand Reinhold Co. NY, 1982.

29.- Herrera P, Valenzuela C. Grupos de estudio pequeños: una búsqueda de evidencias. Medwave 2011; 11 (09). doi: 0.5867/medwave. 2011. 09.5133. 